\title{
Temporary suprapubic diversion in a septic male infant using double-J stent: Indications and surgical technique
}

Daniel T. Keefe; Veridiana Andrioli; Michael P. Leonard

University of Ottawa, Children's Hospital of Eastern Ontario, Ottawa, ON, Canada.

Cite as: Can Urol Assoc J 2018 March 19; Epub ahead of print.

http://dx.doi.org/10.5489/cuaj.5018

Published online March 19, 2018

$* * *$

\section{Introduction}

Infant urethral catheterizations are usually uncomplicated, even with underlying urologic pathology. When catheterization is unsuccessful, surgical intervention may be necessary. The approach for urinary drainage is based on the patient's clinical status, prognosis and future definitive surgical approach.

We present a 9-week-old male infant admitted with urosepsis and renal failure, who had prior unsuccessful catheterization attempts, whereby temporary urinary drainage was achieved with suprapubic placement of a double-J stent. To our knowledge, this is the first description of this technique in the literature.

\section{Case report}

A nine-week-old term male infant presented to a community hospital with fever, reduced intake and decreased urine output. Initial serum creatinine was $30 \mu \mathrm{mol} / \mathrm{L}$ but increased to $90 \mu \mathrm{mol} / \mathrm{L}$ with development of a palpable abdominal mass. A renal bladder ultrasound revealed bilateral hydroureteronephrosis and thick-walled bladder. Blood and urine cultures revealed Klebsiella pneumoniae and Escherischia coli. Antibiotics were initiated. Urethral catheterization was unsuccessful. Transfer to a tertiary hospital was arranged. Serum creatinine on arrival was $167 \mu \mathrm{mol} / \mathrm{L}$ and there was associated anasarca, hypertension and hyperkalemia. Unsuccessful urethral catheterization with a 5Fr feeding tube and a 6Fr catheter with curved tip was attempted by the pediatric urology team. We suspected false passage creation from multiple prior attempts at urethral catheterization.

Urgent urinary bladder drainage was required, however, we felt he was not in optimal clinical condition to tolerate a general anesthetic. We proceeded with temporary suprapubic urinary drainage with a percutaneous double-J stent.

\section{Technique description}


The infant was brought to the radiology department. Procedural sedation was accomplished with ketamine. We marked 1-2 fingerbreadths above the pubic symphysis and prepped and draped this region. Guided by ultrasound, a 16 gauge angiocatheter was inserted into the bladder and urine was aspirated. The needle was removed leaving the angiocatheter in place. A 0.018-inch guidewire was passed through the angiocatheter into the bladder. The angiocatheter was backloaded from the wire. A small incision in the skin was made. A Sof-Flex ${ }^{\circledR} 3.0 \mathrm{Fr}$ by $8 \mathrm{~cm}$ Pediatric Pigtail Ureteral Stent was advanced over the wire and correct position was confirmed by ultrasound. The wire was removed and urine drained from the double-J stent (Fig. 1).

Significant post-obstructive diuresis resulted. Renal function and electrolytes normalized within 48 hours. He was brought to the OR two days post double-J stent insertion for vesicostomy. A VCUG was completed through the vesicostomy confirming the diagnosis of posterior urethral valves (PUV).

\section{Discussion}

PUV are commonly managed with urethral catheterization and subsequent endoscopic valve ablation. In rare cases where urethral catheterization is unsuccessful and patients are medically unfit for immediate surgical intervention, temporary urinary diversion is required to optimize renal function, acquire source control for infections and treat associated electrolyte disturbances. In this case, we report on the delayed presentation of PUV in a 9-week-old boy who presented with urosepsis, renal failure and bladder outlet obstruction. The inability to obtain bladder drainage via urethral catheterization prevented us from proceeding with VCUG to confirm our suspicion of PUV. We felt immediate invasive procedures could risk acute intraoperative and postoperative complications in the setting of renal failure and urosepsis. Suprapubic catheterization was an option however, we felt the SP catheter might be too large for the small infant and be too invasive to complete without general anesthesia.

Prenatal identification of bladder outlet obstruction with ultrasound has allowed in utero intervention to achieve bladder decompression. One method is vesicoamniotic shunting whereby a double pigtail stent (e.g., Rocket KCH Fetal Bladder Drain) is inserted into the fetal bladder percutaneously using a trocar ${ }^{1}$. We extrapolated from this in utero technique in our patient. A suprapubic double-J stent was placed under sedation with ultrasound guidance. The intravesical pigtail prevented dislodging of the suprapubic stent and kept the bladder wall intact for subsequent vesicostomy. Successful urinary drainage was established allowing the patient to become more fit to tolerate a general anesthetic. Previously, Penna et al. (2015) have shown good success from urethral double-J stent placement however this was not an option as urethral access was not achievable in this case ${ }^{2}$. To our knowledge, this is the first use of a suprapubic double $\mathrm{J}$ stent for this indication. 


\section{Conclusion}

Temporary suprapubic urinary diversion with a double-J stent is an effective minimally invasive approach to obtain bladder drainage in infants with bladder outlet obstruction when urethral catheterization is unsuccessful and the patient is not stable enough for definitive surgical intervention.

\section{References}

1. Farrugia M-K: Fetal bladder outlet obstruction: Embryopathology, in utero intervention and outcome. J. Pediatr. Urol. 2016; 12: 296-303.

2. Penna FJ, Bowlin P, Alyami F, et al: Novel Strategy for Temporary Decompression of the Lower Urinary Tract in Neonates Using a Ureteral Stent. J. Urol. 2015; 194: 1086-1090. 


\section{Figures and Tables}

Fig 1. (A) Transverse ultrasound image showing angiocatheter placement within the distended urinary bladder. (B) Post-procedure transverse ultrasound image with arrow showing double-J stent coiling within the urinary bladder. (C) Post-procedure photo showing the suprapubic double-J stent coiling extra-corporeally.

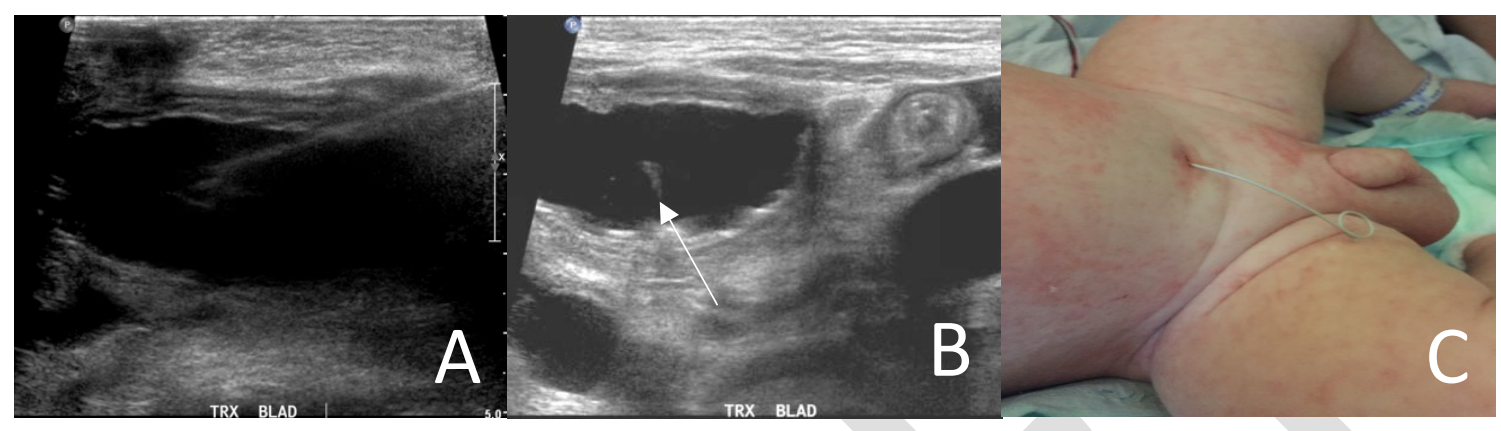

